\title{
Adjuvant interferon for early or late recurrence of hepatocellular carcinoma and mortality from hepatocellular carcinoma following curative treatment: A meta-analysis with comparison of different types of hepatitis
}

\author{
WEI ZHANG ${ }^{1}$, TIAN-QIANG SONG ${ }^{1}$, TI ZHANG ${ }^{1}$, QIANG WU ${ }^{1}$, \\ DA-LU KONG ${ }^{1}$, QIANG LI ${ }^{1}$ and HUI-CHUAN SUN ${ }^{2}$

\begin{abstract}
${ }^{1}$ Key Laboratory of Cancer Prevention and Therapy, Department of Hepatobiliary Surgery, Tianjin Medical University Cancer Institute and Hospital, National Clinical Research Center for Cancer, Tianjin 300060;

${ }^{2}$ Key Laboratory for Carcinogenesis and Cancer Invasion, Liver Cancer Institute and Zhongshan Hospital, Fudan University, The Chinese Ministry of Education, Shanghai 200032, P.R. China
\end{abstract}

Received April 28, 2014; Accepted July 30, 2014

DOI: $10.3892 /$ mco.2014.386

\begin{abstract}
Adjuvant interferon (IFN) therapy following curative treatment for hepatocellular carcinoma (HCC) has been extensively investigated; however, the clinical benefits with different hepatitis backgrounds remain unclear. Medline, Embase, PubMed and the Cochrane Library databases were searched to identify randomized trials and cohort studies that enrolled HCC patients who received curative surgery or ablation therapy followed by IFN and control subjects; the studies were required to include data on early or late recurrence and mortality rates of HCC. Hepatitis B virus (HBV) associated with HCC (HBV-HCC) and hepatitis $\mathrm{C}$ virus (HCV) associated with $\mathrm{HCC}$ (HCV-HCC) were separately analyzed and recurrence, mortality and clinicopathological factors were compared. A total of 14 studies ( 9 randomized trials and 5 cohort studies, including 1,385 patients in total) were eligible for meta-analysis. IFN was found to decrease mortality and early recurrence rates, but exerted no effect on late recurrence rate. The effect of IFN differed between HBV-HCC and HCV-HCC cases. In HCV-HCC, IFN significantly reduced mortality as well as recurrence rates. However, in HBV-HCC patients, IFN
\end{abstract}

Correspondence to: Dr Hui-Chuan Sun, Key Laboratory for Carcinogenesis and Cancer Invasion, Liver Cancer Institute and Zhongshan Hospital, Fudan University, The Chinese Ministry of Education, 136 Yi Xue Yuan Road, Shanghai 200032, P.R. China

E-mail: sun.huichuan@zs-hospital.sh.cn

Dr Wei Zhang, Key Laboratory of Cancer Prevention and Therapy, Department of Hepatobiliary Surgery, Tianjin Medical University Cancer Institute and Hospital, 24 Bin Shui Road, Tianjin 300060, P.R. China

E-mail: zhangweitjch@163.com

Key words: hepatitis B, hepatitis C, hepatocellular carcinoma, interferon, meta-analysis reduced mortality rather than recurrence rates, although it also reduced the recurrence rate in certain subgroups. In conclusion, the effect of adjuvant IFN on postoperative recurrence differed between HBV-HCC and HCV-HCC cases; therefore, different strategies with adjuvant IFN should be used to treat HCC with different hepatitis backgrounds.

\section{Introduction}

Hepatocellular carcinoma (HCC) is one of the leading causes of cancer-related mortality worldwide, with a continuously increasing incidence (1). Surgery and ablation and liver transplantation are the only potentially curative treatments for HCC; however, liver transplantation is restricted by a shortage of liver donors, with surgery and ablation currently being the mainstay of treatment. The tumor recurrence rate is high following liver resection or tumor ablation. Postoperative chemoprevention with vitamin E or vitamin K2 and intervention with ${ }^{131} \mathrm{I}$ are used to reduce tumor recurrence following curative treatment (2); however, there is currently no widely accepted effective adjuvant therapy.

Recurrence may be classified as early, with 1 (3) or 2 years (4) as the cut-off point, or late recurrence (5). Early recurrence may be associated with dissemination of the primary $\mathrm{HCC}$, whereas late recurrence mainly results from de novo tumors arising from the 'field effect' in the diseased liver $(6,7)$. Variables associated with metastasis are associated with early recurrence, whereas those associated with increased carcinogenesis contribute to late recurrence (8). Antitumor treatment may be important in reducing early recurrence, whereas pre- and postoperative antiviral and anti-inflammatory treatments may be crucial in reducing both early and late recurrence (9). The recurrence patterns differ between hepatitis B virus (HBV) associated with HCC (HBV-HCC) and hepatitis $\mathrm{C}$ virus (HCV) associated with $\mathrm{HCC}$ (HCV-HCC) cases. $\mathrm{HBV}-\mathrm{HCC}$ tends to involve more early recurrence due to micrometastases not identified during surgery and HCV-HCC 
tends to involve multicentric late recurrence arising from the cirrhotic liver. The mechanisms of carcinogenesis also differ between $\mathrm{HBV}-\mathrm{HCC}$ and $\mathrm{HCV}-\mathrm{HCC}$; in $\mathrm{HBV}$ patients, $\mathrm{HCC}$ may occur without cirrhosis, whereas cirrhosis is consistently present in patients with HCV-HCC (10). Therefore, multistep and multicentric $\mathrm{HCC}$ develops most frequently in patients with HCV infection (11).

Interferons (IFNs) are cytokines possessing a variety of biological properties, including antiviral, immunomodulatory, antiproliferative and antiangiogenic effects $(12,13)$. IFN is effective in suppressing the replication of HBV and was the first agent to be approved for the treatment of chronic HBV infection (13). IFN has long been used for HCV infection and has effectively decreased progression to liver cirrhosis and development of HCC (14). However, the use of IFN following curative treatment for HCC was not found to be consistently effective and IFN is not considered as the standard adjuvant treatment for HCC following curative treatment. Previous meta-analyses demonstrated that IFN decreased disease recurrence and mortality and prolonged recurrence-free survival (15-17); however, no study has compared the different efficacies of IFN in HBV-HCC and HCV-HCC. The present study focuses on the effect of adjuvant IFN therapy on early and late recurrence of $\mathrm{HCC}$ with different hepatitis backgrounds.

\section{Materials and methods}

Databases and searches. An electronic search was conducted through Medline, Embase, the Cochrane Central Register of Controlled Trials (Central) and the Database of Abstracts of Reviewers of Effectiveness (DARE). The search was limited to the period between January, 1998 and June, 2013, as IFN had not been approved for the treatment of viral hepatitis prior to the late 1990s. 'Hepatocellular carcinoma', 'interferon', 'adjuvant', 'resection and ablation' and 'recurrence' were used as keywords. All the included studies were also entered in the PubMed 'related articles' application and the Science Citation Index to cross-search for similarly indexed studies. Additional studies were identified from article citations.

Data extraction and synthesis. The literature search, data extraction, evaluation and summary were performed independently. Any disagreement was resolved through discussion. The primary efficacy indicator was mortality rate between the IFN treatment and the control groups, whereas the second indicator was recurrence rate, including early and late recurrence. Data regarding the following potential prognostic factors, if available, were extracted from the published reports: patient age, etiology of underlying liver disease (HBV or $\mathrm{HCV}$ ), types of curative therapy (surgery or ablation), tumor characteristics, liver cirrhosis and type, dosage and duration of IFN treatment, including cumulative dosage and average dosage per week. We used early and late recurrence to evaluate the effect of IFN on the tumor or the liver. Since early recurrence was defined as 1 or 2 years postoperatively, we analyzed both 1- and 2-year recurrence; late recurrence was defined as recurrence occurring after 2 years. As HCC mainly develops in hepatitis virus-infected liver, we performed a comparison between studies that enrolled HBV-HCC and those that enrolled HCV-HCC patients.

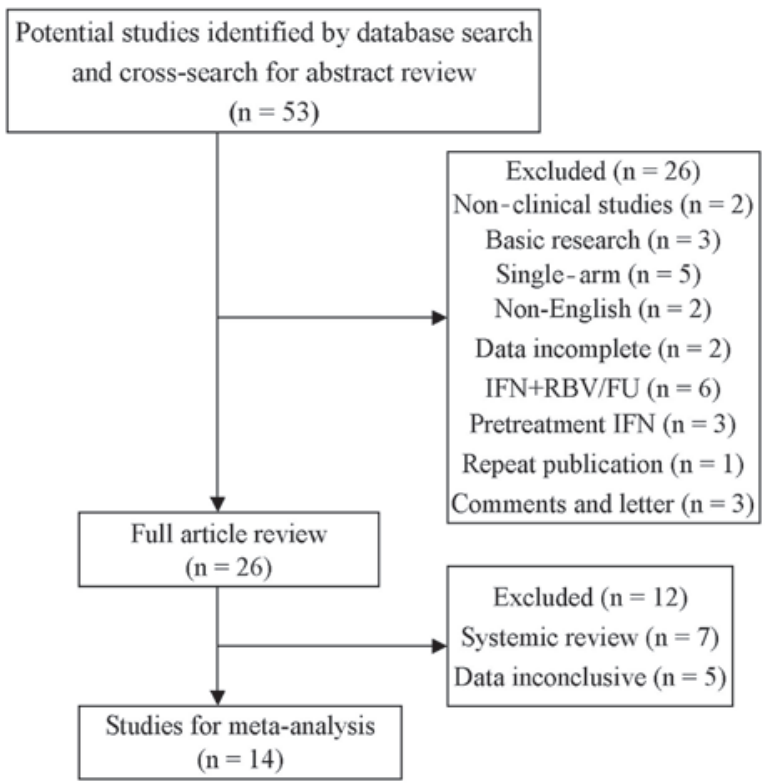

Figure 1. Study flowchart of the data extraction process and selection of studies for meta-analysis. IFN, interferon; RBV, ribavirin; FU, fluorouracil.

Statistical analysis. Meta-analyses were conducted using the fixed-effects and random-effects models and treatment effects were expressed as risk ratios (RRs) with corresponding 95\% confidence intervals ( $95 \%$ CIs). If the fixed- and random-effects models produced the same result, only the fixed-effects estimate was reported. Heterogeneity was assessed using the $\chi^{2}$ statistic and the proportion of variation attributable to heterogeneity was expressed as $\mathrm{I}^{2}$. In the presence of significant heterogeneity $\left(\mathrm{P}<0.05\right.$ for $\left.\chi^{2}\right)$, defined sources of heterogeneity were used, which included the type of IFN ( $\alpha$ or $\beta$ ), type of viral hepatitis (HCV or HBV) and other factors. All the analyses were conducted with Review Manager 5.2 software (Cochrane Collaboration, Oxford, UK).

\section{Results}

Study selection and characteristics. The data extraction process and the selection of studies are outlined in Fig. 1. We also assessed all the systemic reviews on IFN as adjuvant therapy for HCC following curative treatment and studies that were not initially enrolled were added. Finally, we included 14 studies, namely 9 randomized controlled trials (RCT) and 5 case-control studies, including a total of 1,385 patients, comparing the recurrence and mortality rates of $\mathrm{HCC}$ following curative treatment, including resection $(n=5)$, ablation $(n=5)$, or resection plus ablation $(n=4)$.

The characteristics of the studies are summarized in Tables I and II. The sample size for each study varied between 20 and 268 patients, with the median age varying between 49 and 68.5 years. IFN treatment included IFN $\alpha-2 a$, IFN $\alpha-2 b$, IFN $\alpha-1 b$, IFN $\beta$ and pegylated IFN (PEG-IFN). The median tumor size varied between 1.5 and $5.7 \mathrm{~cm}$ and the proportion of multiple tumors varied between 0.0 and $43.8 \%$. The incidence of vessel invasion varied between 19.7 and $76.2 \%$ and the proportion of cirrhosis varied between 47.0 and $100.0 \%$. The median follow-up time was 25-85 months (Table II). 
Table I. Study characteristics and interferon regimens.

\begin{tabular}{|c|c|c|c|c|c|c|c|c|c|c|c|}
\hline $\begin{array}{l}\text { Author } \\
\text { (year) }\end{array}$ & $\begin{array}{l}\text { Patient } \\
\text { number } \\
\text { (IFN/Ctrl) }\end{array}$ & $\begin{array}{l}\text { Study } \\
\text { type }\end{array}$ & $\begin{array}{l}\text { Primary } \\
\text { treatment }\end{array}$ & $\begin{array}{c}\text { Viral } \\
\text { hepatitis }\end{array}$ & Type & IFN schedule & $\begin{array}{l}\text { Cum. } \\
\text { dose } \\
\text { (MU) }\end{array}$ & Weeks & $\begin{array}{l}\text { Dose/ } \\
\text { week } \\
\text { (MU) }\end{array}$ & $\begin{array}{l}\text { Median } \\
\text { follow-up } \\
\quad(\mathrm{mo})\end{array}$ & (Refs.) \\
\hline $\begin{array}{l}\text { Ikeda et al } \\
(2000)\end{array}$ & $10 / 10$ & $\mathrm{RCT}$ & $\mathrm{S} / \mathrm{A}$ & $\mathrm{HCV}$ & $\operatorname{IFN} \beta$ & $6 \mathrm{MU}$ biw x $36 \mathrm{wk}$ & 432 & 36 & 12 & $25 / 25$ & (27) \\
\hline $\begin{array}{l}\text { Kubo et al } \\
(2002)\end{array}$ & $15 / 15$ & $\mathrm{RCT}$ & Surgery & $\mathrm{HCV}$ & $\mathrm{IFN} \alpha$ & $\begin{array}{l}6 \text { MU qd x } 2 \text { wk+ } \\
6 \text { MU tiw x } 14 \text { wk+ } \\
6 \text { MU biw x } 88 \text { wk }\end{array}$ & 1,392 & 104 & 13 & $36 / 30$ & $(22)$ \\
\hline $\begin{array}{l}\text { Suou et al } \\
(2001)\end{array}$ & $18 / 22$ & $\mathrm{CS}$ & $\mathrm{S} / \mathrm{A}$ & $\mathrm{HCV}$ & $\mathrm{IFN} \alpha$ & $\begin{array}{l}6 \mathrm{MU} \text { qd x } 2 \mathrm{wk}+ \\
6 \mathrm{MU} \text { tiw x } 22 \mathrm{wk}\end{array}$ & 480 & 24 & 20 & NA/NA & (37) \\
\hline $\begin{array}{l}\text { Miyaguchi } \\
\text { et al (2002) }\end{array}$ & $16 / 16$ & RCT & $\begin{array}{l}\text { TACE+ } \\
\text { PEI }\end{array}$ & $\mathrm{HCV}$ & $\operatorname{IFN} \alpha-2 b$ & 3 MU tiw x 16 wk & 104 & 16 & 7 & NA & (38) \\
\hline $\begin{array}{l}\text { Shiratori } \\
\text { et al (2003) }\end{array}$ & $49 / 25$ & RCT & Ablation & $\mathrm{HCV}$ & $\mathrm{IFN} \alpha$ & $6 \mathrm{MU}$ tiw x 48 wk & 864 & 48 & 18 & $85 / 85$ & (39) \\
\hline $\begin{array}{l}\text { Lin et al } \\
(2007)\end{array}$ & $20 / 10$ & RCT & Ablation & $\begin{array}{l}\operatorname{HBV}(17) / \\
\operatorname{HCV}(13)\end{array}$ & IFN $\alpha-2 b$ & $\begin{array}{l}3 \mathrm{MU} \text { tiw } \mathrm{x}>24 \mathrm{wk} \\
\text { or } 3 \times 10 / \mathrm{mo} \text { x } 6 \mathrm{mo}+ \\
3 \times 10 / 3 \mathrm{mo} \times 18 \mathrm{mo}\end{array}$ & $\begin{array}{c}212 / \\
360\end{array}$ & $24 / 96$ & $9 / 4$ & $27 / 27$ & (14) \\
\hline $\begin{array}{l}\text { Chen et al } \\
(2012)\end{array}$ & $133 / 135$ & $\mathrm{RCT}$ & Surgery $\mathrm{I}$ & $\begin{array}{l}\operatorname{HBV}(215) / \\
\operatorname{HCV}(53)\end{array}$ & / IFN $\alpha-2 b$ & $\begin{array}{l}1-5 / 1 \text { wk+5 qd x } 4 \text { wk+ } \\
5 \text { MU tiw x } 48 \text { wk }\end{array}$ & 835 & 53 & 16 & $63.8 / 63.8$ & (19) \\
\hline $\begin{array}{l}\text { Mazzaferro } \\
\text { et al (2006) }\end{array}$ & $76 / 74$ & $\mathrm{RCT}$ & Surgery & $\mathrm{HCV}$ & IFN $\alpha-2 b$ & 3 MU tiw x 48 wk & 432 & 48 & 9 & $45 / 45$ & (4) \\
\hline $\begin{array}{l}\text { Sun et al } \\
(2006)\end{array}$ & $118 / 118$ & RCT & Surgery & $\mathrm{HBV}$ & IFN $\alpha-1 b$ & $\begin{array}{l}5 \text { MU qd x 2+ } \\
\text { tiw x } 72 \text { wk }\end{array}$ & 1,092 & 74 & 15 & $36.5 / 36.5$ & (18) \\
\hline $\begin{array}{l}\text { Lo et al } \\
(2007)\end{array}$ & $40 / 40$ & RCT & Surgery & $\begin{array}{l}\operatorname{HBV}(77) / \\
\operatorname{HCV}(3)\end{array}$ & IFN $\alpha-2 b$ & $\begin{array}{c}10 \mathrm{MU} / \mathrm{m}^{2} \text { tiw x } 16 \mathrm{wk} \\
\text { or } 30 \mathrm{MU} / \mathrm{m}^{2} \text { tiw x } 16 \mathrm{wk}\end{array}$ & $\begin{array}{l}480 / \\
1,440\end{array}$ & $16 / 16$ & $30 / 90$ & $>36 />36$ & (13) \\
\hline $\begin{array}{l}\text { Jeong et al } \\
(2007 a)\end{array}$ & $42 / 42$ & CS & $\mathrm{S} / \mathrm{A}$ & $\mathrm{HCV}$ & $\mathrm{IFN} \alpha$ & $\begin{array}{l}6 \mathrm{MU} \text { qd x } 2 \mathrm{wk}+ \\
6 \mathrm{MU} \text { tiw x } 22 \mathrm{wk}\end{array}$ & 480 & 24 & 20 & $37 / 37$ & $(40)$ \\
\hline $\begin{array}{l}\text { Jeong et al } \\
(2007 \mathrm{~b})\end{array}$ & $16 / 16$ & $\mathrm{CS}$ & S/A & $\mathrm{HCV}$ & $\mathrm{IFN} \alpha$ & 3 MU tiw x 48 wk & 432 & 48 & 9 & $32 / 31$ & (20) \\
\hline $\begin{array}{l}\text { Kudo et al } \\
(2007)\end{array}$ & $43 / 84$ & $\mathrm{CS}$ & Ablation & $\mathrm{HCV}$ & $\begin{array}{l}\text { IFN } \alpha-2 b / \\
\text { PEG- } \\
\text { IFN } \alpha-2 a\end{array}$ & $\begin{array}{c}\text { Maintenance } \\
3 \mathrm{MU} / 90 \mu \mathrm{g} \\
\text { qw/q2wk }\end{array}$ & NA & NA & NA & NA & (21) \\
\hline $\begin{array}{l}\text { Hagihara } \\
\text { et al (2011) }\end{array}$ & $37 / 145$ & CS & $\mathrm{S} / \mathrm{A}$ & $\mathrm{HCV}$ & $\begin{array}{c}\text { PEG- } \\
\text { IFN } \alpha-2 \mathrm{a} / 2\end{array}$ & $\begin{array}{c}90-180 \mu \mathrm{g} \mathrm{qw} \times 24-48 \mathrm{wk} \\
(60-100 \mu \mathrm{g} \mathrm{qw} \\
\times 24-48 \mathrm{wk})\end{array}$ & NA & 48 & NA & $44 / 44$ & (41) \\
\hline
\end{tabular}

IFN, interferon; Ctrl, control; cum., cumulative; wk, week; mo, months; RCT, randomized controlled trial; S/A, surgery/ablation; HCV, hepatitis C virus; MU, million units; biw, biweekly; qd, once a day; tiw, three times per week; qw, once per week; q2wk, once every 2 weeks; CS, cohort study; TACE, transarterial chemoembolization; PEI, percutaneous ethanol injection; HBV, hepatitis B virus; NA, not available; PEG-IFN, pegylated IFN

IFN regimen. A total of 12 studies used IFNa (6 studies used IFN $\alpha-2 b, 1$ used IFN $\alpha-1 b$ and 5 used what was only referred to as IFN $\alpha$ ), 1 study used IFN $\beta, 2$ used PEG-IFN $\alpha-2 a$ and 1 used PEG-IFN $\alpha-2 b$. The schedules of IFN administration were also distinct, ranging between 1 and 3 times per week. The cumulative dosage of IFN varied between 104 and 1,440 million units (MU), the duration of IFN treatment varied from 16 to 104 weeks and some studies used IFN without discontinuation. The average dosage per week varied between 4 and 90 MU (Table I).

Treatment efficacy. In all the studies investigating HBV-HCC and $\mathrm{HCV}-\mathrm{HCC}$, we analyzed mortality rates and 1- and 2 -year recurrence rates. For mortality rate analysis, data from one study were unavailable; therefore, a total of 14 studies with 1,385 patients were finally included. IFN treatment significantly reduced the risk of mortality (pooled $\mathrm{RR}=0.60$; 95\% CI: 0.50-0.71; $\mathrm{P}<0.00001)$ (Fig. 2A). The statistical heterogeneity $\left(\mathrm{I}^{2}=54 \% ; \mathrm{P}=0.01\right.$ for $\left.\chi^{2}\right)$ may be partly explained by the study of Chen et al (19), which is the only study reporting that IFN did not reduce the mortality rate for HCC. When this study was excluded from the analysis, the heterogeneity was not statistically significant $\left(\mathrm{I}^{2}=36 \%\right.$; $\mathrm{P}=0.10$ for $\chi^{2}$ ) (data not shown) and the efficacy of IFN was similar among studies.

HCC recurrence was classified as early and late, with the cut-off point for early recurrence varying between 1 and 2 years. We analyzed early (1- and 2-year) and late recurrence 
Table II. Tumor characteristics and patient' follow-up in the interferon treatment/control groups.

\begin{tabular}{|c|c|c|c|c|c|c|c|c|c|c|c|c|}
\hline $\begin{array}{l}\text { Author } \\
\text { (year) }\end{array}$ & $\begin{array}{l}\text { Median } \\
\text { age } \\
\text { (years) }\end{array}$ & $\begin{array}{c}\text { Median } \\
\text { size } \\
(\mathrm{cm})\end{array}$ & $\begin{array}{l}\text { Multiple } \\
(\%)\end{array}$ & $\begin{array}{c}\text { Vessel } \\
\text { invasion } \\
(\%)\end{array}$ & $\begin{array}{c}\text { Cirrhosis } \\
(\%)\end{array}$ & $\begin{array}{l}\text { Median } \\
\text { follow-up } \\
\text { (months) }\end{array}$ & $\begin{array}{l}\text { 1-year } \\
\text { rec. } \\
\text { (n) }\end{array}$ & $\begin{array}{l}\text { 2-year } \\
\text { rec. } \\
\text { (n) }\end{array}$ & $\begin{array}{c}>2 \text {-year } \\
\text { rec. } \\
\text { (n) }\end{array}$ & $\begin{array}{c}\text { No. } \\
\text { of } \\
\text { deaths }\end{array}$ & $\begin{array}{l}\text { Mortality } \\
\text { rate }(\%)\end{array}$ & (Refs.) \\
\hline $\begin{array}{l}\text { Ikeda et al } \\
(2000)\end{array}$ & $60 / 64.5$ & $2.2 / 2$ & $10 / 10$ & $0 / 0$ & $80 / 90$ & $25 / 25$ & $0 / 5$ & $0 / 7$ & $1 / 0$ & $0 / 0$ & $0.0 / 0.0$ & (27) \\
\hline $\begin{array}{l}\text { Kubo et al } \\
(2002)\end{array}$ & $61.9 / 60$ & $2.5 / 2.6$ & $0 / 0$ & NA & $47 / 53$ & $36 / 30$ & $1 / 3$ & $5 / 6$ & $0 / 6$ & $8 / 10$ & $53.3 / 66.7$ & (22) \\
\hline $\begin{array}{l}\text { Suou et al } \\
(2001)\end{array}$ & $61 / 62$ & $2 / 2.1$ & $0 / 0$ & NA & NA & NA & $1 / 3$ & $2 / 7$ & $2 / 8$ & $0 / 6$ & $0.0 / 27.3$ & (37) \\
\hline $\begin{array}{l}\text { Miyaguchi } \\
\text { et al (2002) }\end{array}$ & $66.2 / 65$ & $2.8 / 2.4$ & $27.2 / 33$ & NA & $100 / 100$ & NA & $3 / 10$ & $3 / 14$ & $1 / 0$ & NA & NA & (38) \\
\hline $\begin{array}{l}\text { Shiratori } \\
\text { et al (2003) }\end{array}$ & $61 / 63$ & $2.2 / 2.3$ & $34.7 / 36$ & NA & $100 / 100$ & $85 / 85$ & $12 / 6$ & $25 / 17$ & $15 / 6$ & $19 / 13$ & $38.8 / 52.0$ & (39) \\
\hline $\begin{array}{l}\text { Lin et al } \\
(2007)\end{array}$ & $61.3 / 59$ & $2 / 2.5$ & $15 / 20$ & NA & $90 / 100$ & $27 / 27$ & $56 / 4$ & $5 / 6$ & $3 / 3$ & $4 / 5$ & $20.0 / 50.0$ & (14) \\
\hline $\begin{array}{l}\text { Chen et al } \\
(2012)\end{array}$ & $50 / 49$ & $3.5 / 3$ & $21.5 / 14.8$ & $30.8 / 24.4$ & $53.8 / 55.2$ & $63.8 / 63.8$ & $37 / 35$ & $51 / 55$ & $27 / 21$ & $43 / 41$ & $32.3 / 30.4$ & (19) \\
\hline $\begin{array}{l}\text { Mazzaferro } \\
\text { et al (2006) }\end{array}$ & $65 / 67$ & $3.4 / 3.2$ & $22.4 / 25.7$ & $19.7 / 23.0$ & $94 / 88$ & $45 / 45$ & $25 / 24$ & $40 / 39$ & $4 / 8$ & $17 / 23$ & $22.4 / 31.1$ & (4) \\
\hline $\begin{array}{l}\text { Sun et al } \\
(2006)\end{array}$ & $52.2 / 50.4$ & $4.3 / 4.9$ & $13.6 / 12.7$ & $76.2 / 75.4$ & $83 / 88.1$ & $36.5 / 36.5$ & $32 / 49$ & $52 / 62$ & $15 / 9$ & $38 / 60$ & $32.2 / 50.8$ & (18) \\
\hline $\begin{array}{l}\text { Lo et al } \\
(2007)\end{array}$ & $52 / 51$ & $5.5 / 5.7$ & $17 / 27$ & $48 / 35$ & $48 / 83$ & $>36 />36$ & $2 / 6$ & $17 / 20$ & $4 / 2$ & $8 / 13$ & $20.0 / 32.5$ & (13) \\
\hline $\begin{array}{l}\text { Jeong et al } \\
\text { (2007a) }\end{array}$ & $62 / 63$ & $2 / 1.5$ & $28.6 / 14.3$ & NA & $70.2 / 75.8$ & $37 / 37$ & $0 / 2$ & $9 / 13$ & $11 / 17$ & $2 / 12$ & $4.8 / 28.6$ & (19) \\
\hline $\begin{array}{l}\text { Jeong et al } \\
\text { (2007b) }\end{array}$ & $68.5 / 67.5$ & $1.5 / 1.8$ & $43.8 / 37.5$ & NA & $100 / 100$ & $32 / 31$ & $2 / 1$ & $7 / 11$ & $1 / 2$ & $1 / 6$ & $6.3 / 37.5$ & (40) \\
\hline $\begin{array}{l}\text { Kudo et al } \\
(2007)\end{array}$ & $65 / 66$ & $1.8 / 1.5$ & $19 / 23$ & NA & $70.2 / 75.8$ & NA & $2 / 6$ & $12 / 13$ & $12 / 17$ & $6 / 37$ & $14.0 / 44.0$ & (21) \\
\hline $\begin{array}{l}\text { Hagihara } \\
\text { et al (2011) }\end{array}$ & $63 / 67$ & $1.8 / 2$ & $28 / 28$ & NA & NA & $44 / 44$ & $5 / 33$ & $17 / 76$ & $1 / 25$ & $2 / 49$ & $5.4 / 33.8$ & (41) \\
\hline
\end{tabular}

NA, not available; rec., recurrence.

(>2 years). A total of 14 studies, including 1,385 patients, were used for the assessment of early and late recurrence and IFN treatment was found to significantly reduce the risk of 1-year recurrence (pooled $\mathrm{RR}=0.76 ; 95 \% \mathrm{CI}$ : 0.62-0.92; $\mathrm{P}=0.005$ ). The heterogeneity was not significant $\left(\mathrm{I}^{2}=3 \% ; \mathrm{P}=0.42\right.$ for $\left.\chi^{2}\right)$ (Fig. 2B).

IFN treatment significantly reduced the risk of 2-year recurrence (pooled $\mathrm{RR}=0.77 ; 95 \% \mathrm{CI}$ : 0.68-0.87; $\mathrm{P}<0.0001$ ). The heterogeneity was not statistically significant $\left(\mathrm{I}^{2}=33 \%\right.$; $\mathrm{P}=0.11$ for $\chi^{2}$ ) (Fig. 2C).

IFN treatment did not significantly reduce the risk of late recurrence (recurrence $>2$ years; pooled $\mathrm{RR}=0.91$; 95\% CI: 0.71-1.17; $\mathrm{P}=0.45)$. The heterogeneity was marginally statistically significant $\left(\mathrm{I}^{2}=35 \% ; \mathrm{P}=0.09\right.$ for $\left.\chi^{2}\right)$ (Fig. 2D).

Subgroup analysis according to type of hepatitis virus. As HBV-HCC and HCV-HCC exhibited different characteristics in the carcinogenesis of $\mathrm{HCC}$, we performed a subgroup analysis according to different types of hepatitis. In studies that enrolled only patients with $\mathrm{HCV}-\mathrm{HCC}$, IFN reduced the mortality and recurrence rates, including both early and late recurrence (Fig. 3). IFN reduced mortality rate (pooled $\mathrm{RR}=0.44$; 95\% CI: $0.34-0.58 ; \mathrm{P}<0.00001)$ and the heterogeneity was not significant $\left(\mathrm{I}^{2}=48 \%\right.$; $\mathrm{P}=0.05$ for $\left.\chi^{2}\right)$ (Fig. 3A). IFN also decreased 1-year early recurrence rate (pooled $\mathrm{RR}=0.69$; 95\% CI: 0.51-0.93; $\mathrm{P}=0.02$ ) (Fig. 3B) and 2-year recurrence rate (pooled $\mathrm{RR}=0.69 ; 95 \% \mathrm{CI}$ : 0.58-0.82; $\mathrm{P}<0.0001$ ) (Fig. 3C). Moreover, late recurrence rate was also significantly decreased (pooled RR=0.68; 95\% CI: 0.49-0.94; $\mathrm{P}=0.02$ ) (Fig. 3D).

In studies enrolling only patients with HBV-HCC, IFN only reduced the mortality rate (pooled $\mathrm{RR}=0.79$; 95\% CI: 0.63-0.98; $\mathrm{P}=0.03$ ). However, early recurrence rates were not significantly reduced at 1-year (pooled $\mathrm{RR}=0.82 ; 95 \% \mathrm{CI}$ : 0.64-1.05; $\mathrm{P}=0.12$ ) or at 2-years (pooled $\mathrm{RR}=0.89 ; 95 \% \mathrm{CI}$ : 0.74-1.08; $\mathrm{P}=0.24$ ). Additionally, the late recurrence rate was not reduced with IFN treatment (pooled RR=1.45; 95\% CI: 0.96-2.20; $\mathrm{P}=0.08$ ) (Fig. 4). 
A

\begin{tabular}{|c|c|c|c|c|c|c|c|c|}
\hline Study or Subgroup & \multicolumn{2}{|c|}{ Interferon } & \multicolumn{2}{|c|}{ Control } & \multicolumn{2}{|r|}{$\begin{array}{l}\text { Risk Ratio } \\
\text { Cl Fixed } 95 \% \text { Year }\end{array}$} & \multicolumn{2}{|c|}{$\begin{array}{c}\text { Risk Ratio } \\
\text { M-H, Fixed, 95\% Cl }\end{array}$} \\
\hline Ikeda K 2000 & 0 & 10 & 0 & 10 & & Not estimable 2000 & & \\
\hline Suou T 2001 & 0 & 18 & 6 & 22 & $2.4 \%$ & $0.09[0.01,1.55] 2001$ & & \\
\hline Miyaguchi S 2002 & 5 & 16 & 14 & 16 & $5.6 \%$ & $0.36[0.17,0.76] 2002$ & - & \\
\hline Kubo S 2002 & 8 & 15 & 10 & 15 & $4.0 \%$ & $0.80[0.44,1.45] 2002$ & $\rightarrow$ & \\
\hline Shiratori Y 2003 & 19 & 49 & 13 & 25 & $6.9 \%$ & $0.75[0.45,1.25] 2003$ & 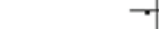 & \\
\hline Lin SM 2007 & 4 & 20 & 5 & 10 & $2.7 \%$ & $0.40[0.14,1.17] 2003$ & & \\
\hline Sun $\mathrm{HC} 2006$ & 38 & 118 & 60 & 118 & $24.0 \%$ & $0.63[0.46,0.87] 2006$ & \#- & \\
\hline Mazzaferro V 2006 & 17 & 76 & 23 & 74 & $9.3 \%$ & $0.72[0.42,1.23] 2006$ & $\rightarrow$ & \\
\hline Lo CM 2007 & 8 & 40 & 13 & 40 & $5.2 \%$ & $0.62[0.29,1.32] 2007$ & . & \\
\hline Jeong S 2007b & 1 & 16 & 6 & 16 & $2.4 \%$ & $0.17[0.02,1.23] 2007$ & & \\
\hline Kudo M 2007 & 6 & 43 & 37 & 84 & $10.0 \%$ & $0.32[0.15,0.69] 2007$ & & \\
\hline Jeong S 2007a & 2 & 42 & 12 & 42 & $4.8 \%$ & $0.17[0.04,0.70] 2007$ & & \\
\hline Hagihara H 2011 & 2 & 37 & 39 & 145 & $6.4 \%$ & $0.20[0.05,0.79] 2011$ & & \\
\hline Chen LT 2012 & 43 & 133 & 41 & 135 & $16.3 \%$ & $1.06[0.75,1.52] 2012$ & & \\
\hline Total $(95 \% \mathrm{Cl})$ & & 633 & & 752 & $100.0 \%$ & $0.60[0.50,0.71]$ & $\downarrow$ & \\
\hline Total events & 153 & & 279 & & & & & \\
\hline $\begin{array}{l}\text { Heterogeneity: } \mathrm{Ch}^{2}= \\
\text { Test for overall effect }\end{array}$ & $\begin{array}{l}6.00, d f= \\
=5.91(P\end{array}$ & $\begin{array}{l}12(P= \\
<0.00\end{array}$ & $\begin{array}{l}0.01) ; 1^{2} \\
001)\end{array}$ & $=54 \%$ & & & $\begin{array}{cc}0.01 & 0.1 \\
\text { Favours inte }\end{array}$ & 11 \\
\hline
\end{tabular}

B

$\begin{array}{cccc}\text { Interferon } & \text { Control } & \text { Risk Ratio } \\ \text { Study or Subgroup } & \text { Events Total Events Total Weight } & \text { M-H, Fixed, } 95 \% \text { Cl Year }\end{array}$

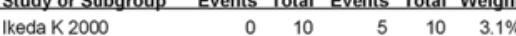
Suou $\mathrm{T} 2001$ Kubo 52002

Miyaguchi S 2002

Lin SM 2007

Shiratori Y 2003

Mazzaferro V 2006

Sun HC 2006

Jeong S 2007b

Kudo M 2007

LO CM 2007

Jeong S 2007a

Hagihara H 2011

Chen LT 2012

Total $(95 \% \mathrm{Cl})$

Total events

$\begin{array}{lllll}0 & 10 & 5 & 10 & 3.1 \% \\ 1 & 18 & 3 & 22 & 1.5 \%\end{array}$

Favours interferon Favours control

Heterogeneity: $\mathrm{Ch}^{2}=13.43, \mathrm{df}=13(\mathrm{P}=0.42) ; \mathrm{I}^{2}=3 \%$

Test for overall effect: $Z=2.80(P=0.005)$

C

$\begin{array}{llll} & \text { Interferon Control } & \text { Risk Ratio } \\ \text { Study or Subgroup } & \end{array}$

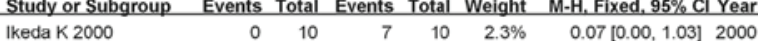
$\begin{array}{llllllll}\text { Ikeda K 2000 } & 0 & 10 & 7 & 10 & 2.3 \% & 0.07[0.00,1.03] & 2000 \\ \text { Suou T 2001 } & 2 & 18 & 7 & 22 & 1.9 \% & 0.35[0.08,1.48] & 2001\end{array}$ $\begin{array}{llllllll}\text { Suou T 2001 } & 2 & 18 & 7 & 22 & 1.9 \% & 0.35[0.08,1.48] & 2001 \\ \text { Kubo S 2002 } & 5 & 15 & 6 & 15 & 1.8 \% & 0.83[0.32,2.15] & 2002\end{array}$

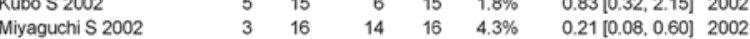
$\begin{array}{llllllll}\text { Shiratori } Y 2003 & 25 & 49 & 17 & 25 & 6.9 \% & 0.75[0.51,1.10] & 2003\end{array}$ $\begin{array}{llllllll}\text { Lin SM } 2007 & 5 & 20 & 6 & 10 & 2.5 \% & 0.42[0.17,1.04] 2003\end{array}$ $\begin{array}{llllllll}\text { Mazzaferro V } 2006 & 40 & 76 & 39 & 74 & 12.2 \% & 1.00[0.74,1.35] & 2006\end{array}$ $\begin{array}{llllllll}\text { Sun HC } 2006 & 52 & 118 & 62 & 118 & 19.1 \% & 0.84[0.64,1.09] & 2006\end{array}$ $\begin{array}{lllllllll}\text { Jeong S 2007b } & 7 & 16 & 11 & 16 & 3.4 \% & 0.64[0.33,121] & 2007\end{array}$ $2 \mathrm{CM} 2007$ $17 \quad 40-20,4026.2 \%[0.85[0.53,1.37] 2007$ $\begin{array}{lllllll} & 12 & 43 & 43 & 84 & 9.0 \% & 0.55[0.32,0.92\end{array} 2007$ $\begin{array}{llrrrrrr}\text { Hagihara H 2011 } & 17 & 37 & 76 & 145 & 9.5 \% & 0.88[0.60,1.28] & 2011 \\ \text { Chen LT 2012 } & 51 & 133 & 55 & 135 & 16.8 \% & 0.94[0.70,1.27] & 2012\end{array}$ $\begin{array}{lllll}\text { Total }(95 \% \mathrm{Cl}) \quad 633 & 752 & 100.0 \% & 0.77 & {[0.68,0.87]}\end{array}$

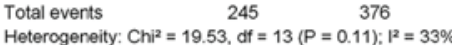
Test for overall effect $Z=4.14(P<0.0001)$

D

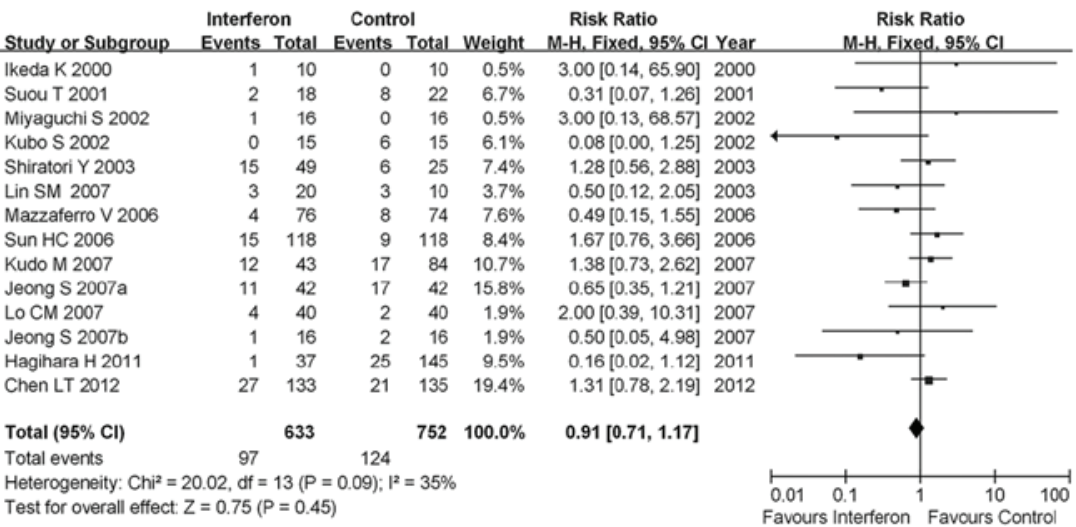

Figure 2. Forest plot of the 14 studies of adjuvant interferon therapy for hepatocellular carcinoma patients following curative therapy. (A) Overall mortality; (B) 1-year early recurrence; (C) 2-year early recurrence; (D) late recurrence. CI, confidence interval.

We further compared the clinicopathological data for HBV-HCC and HCV-HCC patients and found that patients with HBV-HCC had larger tumors, a higher proportion of single tumors and more tumors with vascular invasion; however, these patients had less severe liver cirrhosis, indicating that $\mathrm{HBV}-\mathrm{HCC}$ was more aggressive and that liver cirrhosis in 


\begin{tabular}{|c|c|c|c|c|c|c|c|c|c|c|}
\hline $\mathbf{A}$ & Study or Subgroup & $\begin{array}{l}\text { Interfero } \\
\text { Events }\end{array}$ & $\begin{array}{l}\text { on } \\
\text { Total }\end{array}$ & $\begin{array}{l}\text { Contro } \\
\text { Events }\end{array}$ & $\begin{array}{l}\text { ol } \\
\text { Total }\end{array}$ & Weight & $\begin{array}{l}\text { Risk Ratio } \\
\text { M-H. Fixed. } 95 \% \mathrm{Cl}\end{array}$ & Year & $\begin{array}{r}\text { Risk F } \\
\text { M-H. Fixes }\end{array}$ & $\begin{array}{l}\text { Ratio } \\
\text { d. } 95 \% \mathrm{Cl}\end{array}$ \\
\hline & Ikeda K 2000 & 0 & 10 & 0 & 10 & & Not estimable & 2000 & & \\
\hline & Suou T 2001 & 0 & 18 & 6 & 22 & $4.3 \%$ & $0.09[0.01,1.55]$ & 2001 & & \\
\hline & Kubo S 2002 & 8 & 15 & 10 & 15 & $7.4 \%$ & $0.80[0.44,1.45]$ & 2002 & & \\
\hline & Miyaguchi S 2002 & 5 & 16 & 14 & 16 & $10.3 \%$ & $0.36[0.17,0.76]$ & 2002 & & \\
\hline & Shiratori Y 2003 & 19 & 49 & 13 & 25 & $12.7 \%$ & $0.75[0.45,1.25]$ & 2003 & & \\
\hline & Lin SM 2007 & 4 & 20 & 5 & 10 & $4.9 \%$ & $0.40[0.14,1.17]$ & 2003 & & \\
\hline & Mazzaferro V 2006 & 17 & 76 & 23 & 74 & $17.1 \%$ & $0.72[0.42,1.23]$ & 2006 & & \\
\hline & Jeong S 2007a & 2 & 42 & 12 & 42 & $8.8 \%$ & $0.17[0.04,0.70]$ & 2007 & & \\
\hline & Jeong S 2007b & 1 & 16 & 6 & 16 & $4.4 \%$ & $0.17[0.02,1.23]$ & 2007 & & \\
\hline & Kudo M 2007 & 6 & 43 & 37 & 84 & $18.4 \%$ & $0.32[0.15,0.69]$ & 2007 & & \\
\hline & Hagihara H 2011 & 2 & 37 & 39 & 145 & $11.7 \%$ & $0.20[0.05,0.79]$ & 2011 & & \\
\hline & Total $(95 \% \mathrm{Cl})$ & & 342 & & 459 & $100.0 \%$ & $0.44[0.34,0.58]$ & & $\boldsymbol{\gamma}$ & \\
\hline & Total events & 64 & & 165 & & & & & & \\
\hline & $\begin{array}{l}\text { Heterogeneity: } \mathrm{Chi}^{2}= \\
\text { Test for overall effect: }\end{array}$ & $\begin{array}{l}17.17, d f= \\
Z=6.04(P\end{array}$ & $\begin{array}{l}9(P=C \\
<0.00\end{array}$ & $\begin{array}{l}0.05) ; 1^{2}= \\
0001)\end{array}$ & $=48 \%$ & & & & $\begin{array}{cc}0.01 & 0.1\end{array}$ & \begin{tabular}{|cc}
10 & 100 \\
Favours control
\end{tabular} \\
\hline B & Study or Subgroup & $\begin{array}{l}\text { Interferon } \\
\text { Events } T\end{array}$ & cotal E & $\begin{array}{l}\text { Control } \\
\text { Events } T\end{array}$ & I & Weight & $\begin{array}{c}\text { Risk Ratio } \\
\mathrm{M}-\mathrm{H} \text {. Fixed, } 95 \% \mathrm{Cl}\end{array}$ & Year & $\begin{array}{r}\text { Risk R } \\
\text { M-H. Fixed }\end{array}$ & $\begin{array}{l}\text { Ratio } \\
\text { d. } 95 \% \mathrm{Cl}\end{array}$ \\
\hline & Ikeda K 2000 & 0 & 10 & 5 & 10 & $6.9 \%$ & $0.09[0.01,1.45]$ & 2000 & & \\
\hline & Suou T 2001 & 1 & 18 & 3 & 22 & $3.4 \%$ & $0.41[0.05,3.59]$ & 2001 & & \\
\hline & Kubo S 2002 & 1 & 15 & 3 & 15 & $3.8 \%$ & $0.33[0.04,2.85]$ & 2002 & & \\
\hline & Miyaguchi S 2002 & 3 & 16 & 10 & 16 & $12.5 \%$ & $0.30[0.10,0.89]$ & 2002 & & \\
\hline & Shiratori Y 2003 & 12 & 49 & 6 & 25 & $10.0 \%$ & $1.02[0.43,2.40]$ & 2003 & & \\
\hline & Lin SM 2007 & 5 & 20 & 4 & 10 & $6.7 \%$ & $0.63[0.21,1.83]$ & 2003 & & \\
\hline & Mazzaferro V 2006 & 25 & 76 & 24 & 74 & $30.5 \%$ & $1.01[0.64,1.61]$ & 2006 & & \\
\hline & Jeong S 2007a & 0 & 42 & 2 & 42 & $3.1 \%$ & $0.20[0.01,4.04]$ & 2007 & & \\
\hline & Kudo M 2007 & 2 & 43 & 6 & 84 & $5.1 \%$ & $0.65[0.14,3.09]$ & 2007 & & \\
\hline & $\begin{array}{l}\text { Jeong S 2007b } \\
\text { Hagihara H } 2011\end{array}$ & $\begin{array}{l}2 \\
5\end{array}$ & $\begin{array}{l}16 \\
37\end{array}$ & $\begin{array}{r}1 \\
33\end{array}$ & $\begin{array}{r}16 \\
145\end{array}$ & $\begin{array}{r}1.3 \% \\
16.8 \%\end{array}$ & $\begin{array}{r}2.00[0.20,19.91] \\
0.59[0.25,1.42]\end{array}$ & $\begin{array}{l}2007 \\
2011\end{array}$ & & \\
\hline & Total $(95 \% \mathrm{Cl})$ & & 342 & & 4591 & $100.0 \%$ & $0.69[0.51,0.93]$ & & & \\
\hline & Total events & 56 & & 97 & & & & & & \\
\hline & $\begin{array}{l}\text { Heterogeneity: } \mathrm{Chi}^{2}=1 \\
\text { Test for overall effect: } Z\end{array}$ & $\begin{array}{l}0.13, d f=1 \\
=2.41(P=\end{array}$ & $\begin{array}{l}0(P=C \\
=0.02)\end{array}$ & $0.43) ; 1^{2}=$ & $=1 \%$ & & & & $\begin{array}{lll}0.01 & 0.1 & 1 \\
\text { Favours } & \text { Interferon }\end{array}$ & $\begin{array}{l}10 \quad 100 \\
\text { Favours control }\end{array}$ \\
\hline C & Study or Subgroup & $\begin{array}{l}\text { Interfero } \\
\text { Events }\end{array}$ & $\begin{array}{l}\text { on } \\
\text { Total }\end{array}$ & $\begin{array}{l}\text { Contro } \\
\text { Events }\end{array}$ & Total & Weight & $\begin{array}{c}\text { Risk Ratio } \\
\text { M-H. Fixed. } 95 \% \mathrm{Cl}\end{array}$ & Y Year & $\begin{array}{r}\text { Risk R } \\
\text { M-H. Fixed }\end{array}$ & $\begin{array}{l}\text { Ratio } \\
\text { d. } 95 \% \mathrm{Cl}\end{array}$ \\
\hline & Ikeda K 2000 & 0 & 10 & 7 & 10 & $4.0 \%$ & $0.07[0.00,1.03]$ & 2000 & & \\
\hline & Suou T 2001 & 2 & 18 & 7 & 22 & $3.4 \%$ & $0.35[0.08,1.48]$ & 2001 & & \\
\hline & Miyaguchi S 2002 & 3 & 16 & 14 & 16 & $7.5 \%$ & $0.21[0.08,0.60]$ & 2002 & & \\
\hline & Kubo S 2002 & 5 & 15 & 6 & 15 & $3.2 \%$ & $0.83[0.32,2.15]$ & 2002 & & \\
\hline & Lin SM 2007 & 5 & 20 & 6 & 10 & $4.3 \%$ & $0.42[0.17,1.04]$ & 2003 & & \\
\hline & Shiratori Y 2003 & 25 & 49 & 17 & 25 & $12.0 \%$ & $0.75[0.51,1.10]$ & 2003 & & \\
\hline & Mazzaferro V 2006 & 40 & 76 & 39 & 74 & $21.0 \%$ & $1.00[0.74,1.35]$ & 2006 & & \\
\hline & Jeong S 2007a & 9 & 42 & 13 & 42 & $6.9 \%$ & $0.69[0.33,1.44]$ & 2007 & 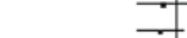 & \\
\hline & Jeong S 2007b & 7 & 16 & 11 & 16 & $5.9 \%$ & $0.64[0.33,1.21]$ & 2007 & $\because$ & \\
\hline & $\begin{array}{l}\text { Kudo M } 2007 \\
\text { Hagihara H } 2011\end{array}$ & $\begin{array}{l}12 \\
17\end{array}$ & $\begin{array}{l}43 \\
37\end{array}$ & $\begin{array}{l}43 \\
76\end{array}$ & $\begin{array}{r}84 \\
145\end{array}$ & $\begin{array}{l}15.5 \% \\
16.4 \%\end{array}$ & $\begin{array}{l}0.55[0.32,0.92] \\
0.88[0.60,1.28]\end{array}$ & $\begin{array}{l}2007 \\
2011\end{array}$ & - & \\
\hline & Total $(95 \% \mathrm{Cl})$ & & 342 & & 459 & $100.0 \%$ & $0.69[0.58,0.82]$ & & $\checkmark$ & \\
\hline & Total events & 125 & & 239 & & & & & & \\
\hline & $\begin{array}{l}\text { Heterogeneity: } \mathrm{Chi}^{2}= \\
\text { Test for overall effect: }\end{array}$ & $\begin{array}{l}18.15, \mathrm{df}= \\
Z=4.32(P\end{array}$ & $\begin{array}{l}10(P= \\
<0.00\end{array}$ & $\begin{array}{l}=0.05) ; 1^{2}= \\
\text { D01) }\end{array}$ & $=45 \%$ & & & & $\begin{array}{ccc}0.01 & 0.1\end{array}$ & Favours control \\
\hline D & Study or Subgroup & $\begin{array}{l}\text { Interferc } \\
\text { Events }\end{array}$ & $\begin{array}{l}\text { on } \\
\text { Total }\end{array}$ & $\begin{array}{l}\text { Contro } \\
\text { Events }\end{array}$ & $\begin{array}{l}\text { ol } \\
\text { Total }\end{array}$ & Weight & $\begin{array}{c}\text { Risk Ratio } \\
\text { M-H. Fixed. } 95 \% \mathrm{C}\end{array}$ & cl Year & $\begin{array}{r}\text { Risk F } \\
\text { M-H. Fixes }\end{array}$ & $\begin{array}{l}\text { Ratio } \\
\text { ed. } 95 \% \mathrm{Cl}\end{array}$ \\
\hline & Ikeda K 2000 & 1 & 10 & 0 & 10 & $0.7 \%$ & $3.00[0.14,65.90]$ & 2000 & & \\
\hline & Suou T 2001 & 2 & 18 & 8 & 22 & $9.5 \%$ & $0.31[0.07,1.26]$ & 2001 & & \\
\hline & Miyaguchi S 2002 & 1 & 16 & 0 & 16 & $0.7 \%$ & $3.00[0.13,68.57]$ & 2002 & & \\
\hline & Kubo S 2002 & $\begin{array}{l}0 \\
3\end{array}$ & 15 & $\begin{array}{l}6 \\
3\end{array}$ & 15 & $8.6 \%$ & $0.08[0.00,1.25]$ & 2002 & & \\
\hline & $\begin{array}{l}\text { Lin SM } 2007 \\
\text { Shiratori Y } 2003\end{array}$ & $\begin{array}{r}3 \\
15\end{array}$ & $\begin{array}{l}20 \\
49\end{array}$ & $\begin{array}{l}3 \\
6\end{array}$ & $\begin{array}{l}10 \\
25\end{array}$ & $\begin{array}{r}5.3 \% \\
10.5 \%\end{array}$ & $\begin{array}{l}0.50[0.12,2.05] \\
1.28[0.56,2.88]\end{array}$ & $\begin{array}{l}2003 \\
2003\end{array}$ & & 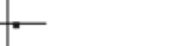 \\
\hline & Mazzaferro V 2006 & 4 & 76 & 8 & 74 & $10.7 \%$ & $0.49[0.15,1.55]$ & 2006 & & - \\
\hline & Kudo M 2007 & 12 & 43 & 17 & 84 & $15.3 \%$ & $1.38[0.73,2.62]$ & 2007 & & - \\
\hline & Jeong S 2007b & 1 & 16 & 2 & 16 & $2.7 \%$ & $0.50[0.05,4.98]$ & 2007 & & \\
\hline & Jeong S 2007a & 11 & 42 & 17 & 42 & $22.5 \%$ & $0.65[0.35,1.21]$ & 2007 & & \\
\hline & Hagihara H 2011 & 1 & 37 & 25 & 145 & $13.5 \%$ & $0.16[0.02,1.12]$ & 2011 & & \\
\hline & Total $(95 \% \mathrm{Cl})$ & & 342 & & 459 & $100.0 \%$ & $0.68[0.49,0.94]$ & & & \\
\hline & Total events & 51 & & 92 & & & & & & \\
\hline & $\begin{array}{l}\text { Heterogeneity: } \mathrm{Ch}^{2}= \\
\text { Test for overall effect: }\end{array}$ & $\begin{array}{l}15.02, d f= \\
Z=2.34(P\end{array}$ & $\begin{array}{l}10(P= \\
=0.02\end{array}$ & $\begin{array}{l}=0.13) ; 1^{2}= \\
\text { 2) }\end{array}$ & $=33 \%$ & & & & $\begin{array}{lll}0.01 & 0.1 & 1\end{array}$ & 1 \\
\hline
\end{tabular}

Figure 3. Forest plot of the 11 studies on adjuvant interferon therapy for hepatitis $\mathrm{C}$ virus associated with hepatocellular carcinoma patients following curative therapy. (A) overall mortality; (B) 1-year early recurrence; (C) 2-year early recurrence; (D) late recurrence. CI, confidence interval.

HBV-HCC was less severe compared to that in HCV-HCC (Table III).

Since IFN did not reduce the recurrence rate in HBV-HCC, we performed further analyses of individual studies and found that in the study by Lo et al (13), IFN significantly reduced the recurrence rate in patients with TNM stage III/IVA, indicating the direct antitumor effect of IFN. In the study by Sun et al (18), IFN significantly prolonged disease-free survival, although the tumor recurrence rate was not decreased. However, in the study by Chen et al (19), IFN neither prolonged survival nor reduced the mortality or recurrence rates, which may be attributable to the obvious bias in that study, with the IFN group exhibiting 
Table III. Comparison of clinicopathological characteristics between HBV-HCC and HCV-HCC cases.

\begin{tabular}{llrr}
\hline Characteristics & Group & HBV-HCC, median (range) & HCV-HCC $^{\mathrm{b}}$, median $^{\text {(range })}$ \\
\hline Tumor size (cm) & IFN & $3.9(2.5-5.5)$ & $2(1.5-3.4)$ \\
& Control & $3.95(2.1-5.7)$ & $2.1(1.5-2.6)$ \\
Multiple tumors (\%) & IFN & $17.5(17-21.5)$ & $27.2(10-43.8)$ \\
& Control & $15.9(12.7-27)$ & $25.7(10-37.5)$ \\
Vascular invasion (\%) & IFN & $48(30.8-76.2)$ & $19.7^{\mathrm{b}}$ \\
Cirrhosis $(\%)$ & Control & $35(24.4-75.4)$ & $23^{\mathrm{b}}$ \\
& IFN & $53.8(48-83)$ & $90(47-100)$ \\
& Control & $83(44.2-88.1)$ & $90(53-100)$
\end{tabular}

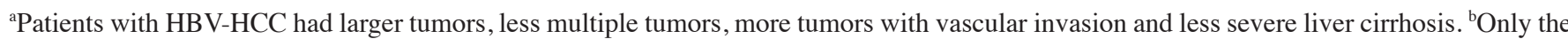
study by Mazzaferro et al (4) was included because information regarding vascular invasion in other studies was unavailable. HBV, hepatitis B virus; $\mathrm{HCC}$, hepatocellular carcinoma; $\mathrm{HCV}$, hepatitis $\mathrm{C}$ virus; IFN, interferon.

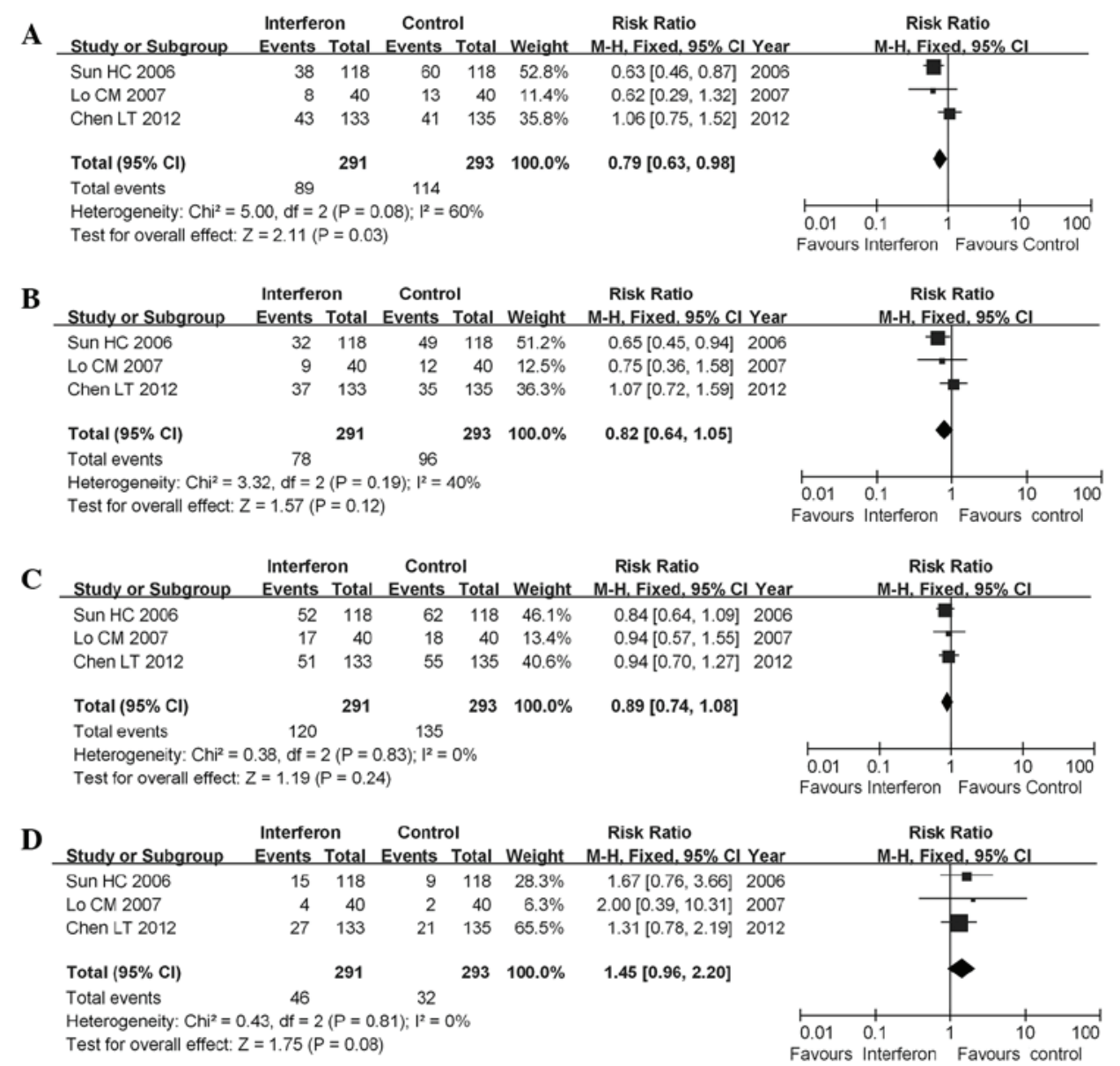

Figure 4. Forest plot of the 3 studies on adjuvant interferon therapy for hepatitis B virus associated with hepatocellular carcinoma patients following curative therapy. (A) overall mortality; (B) 1-year early recurrence; (C) 2-year early recurrence; (D) late recurrence. CI, confidence interval.

more unfavorable tumor characteristics, such as larger tumors (3.5 vs. $3.0 \mathrm{~cm})$, multiple tumors $(21.5$ vs. $14.8 \%)$ and high incidence of microvascular invasion (30.8 vs. $24.4 \%$ ). Liver traits, such as hepatitis activity, were also more severe in the IFN group (hepatitis activity, 63.9 vs. 51.1\%). However, information regarding TNM staging for both groups was unavailable, making subset analysis of TNM staging impossible.
Second recurrence and prognosis of patients with recurrent tumors. The reduction in mortality rate by IFN is associated with its inhibition of recurrence; a number of studies have reported that recurrent tumors after adjuvant IFN therapy are more likely to receive potentially curative treatment. Jeong et al (20) demonstrated that the second recurrence rate was significantly lower in the IFN compared to that in the non-IFN group. 
Kudo et al (21) also demonstrated that, in IFN-treated patients, all the recurrent tumors were single and $<2.0 \mathrm{~cm}$ in diameter and could be completely retreated by radiofrequency ablation in all the cases. However, in the control group, recurrent tumors tended to be multiple and the majority were incurable by local therapy. Kubo et al (22) reported that recurrent tumors in the IFN group were mostly single, but in the control group multiple tumors were more common. Sun et al (18) demonstrated that the median overall survival rate of patients with tumor recurrence was longer in the IFN $\alpha$ compared to that in the control group.

Bias analysis. One study used IFN $\beta$, whereas other studies exclusively used IFN $\alpha$, including IFN $\alpha$ in 6 , IFN $\alpha-2 b$ in 6 , IFN $\alpha-2 \mathrm{a}$ in 2, IFN $\alpha-1 \mathrm{~b}$ in 1 and PEG-IFN $\alpha$ in 2 studies. The cumulative dosage varied between 104 and 1,440 MU, the duration of the IFN treatment varied from 4 months to 2 years. We also calculated dosage per week, which varied 4-90 MU/week. The withdrawal rate varied between 0 and $17.1 \%$ and adverse effects were common in the IFN group. We did not analyze side effects, due to the heterogeneity of the data. In the study by Mazzaferro et al (4), only 28 of the 76 patients in the IFN group were adherent to the protocol according to definition, reflecting poor compliance.

Other systemic reviews or meta-analyses. Breitenstein etal (23) reported that IFN significantly reduced the 2-year mortality rate following curative treatment of $\mathrm{HCC}$ (pooled $\mathrm{RR}=0.65$; 95\% CI: $0.52-0.80 ; \mathrm{P}<0.001)$ in the absence of any significant heterogeneity $\left(\mathrm{I}^{2}=0 \% ; \mathrm{P}=0.823\right.$ for $\left.\chi^{2}\right)$. The effect on reduction of tumor recurrence was less pronounced, but still significant (pooled $\mathrm{RR}=0.86$; 95\% CI: 0.76-0.97; $\mathrm{P}=0.013$ ). Zhang et al (24) reported that IFN significantly reduced early recurrence (1-1.5 years after surgery) and 1-year survival. Zhong et al (2) reported that IFN significantly reduced 2 -year recurrence and 2-year overall survival. Shen et al (16) demonstrated that adjuvant IFN prolonged 1-, 2- and 3-year recurrence-free survival rates of patients with HCC. However, all the meta-analyses included patients with HBV-HCC and patients with HCV-HCC and stratification according to different types of hepatitis was lacking.

\section{Discussion}

The present study systematically reviewed the published RCTs and cohort studies on adjuvant IFN therapy for HCC and found that IFN significantly reduced the mortality and early recurrence rates (1- and 2-year) of HCC following curative treatment; however, IFN treatment did not reduce late ( $>2$ years) recurrence. Furthermore, the effect of IFN on HCC with a different hepatitis background varied. In HCV-HCC cases, IFN reduced the mortality and recurrence rates, including both early and late recurrence. However, in HBV-HCC cases, IFN only reduced the mortality rate, whereas neither early nor late recurrence rates were reduced. Further analysis demonstrated that tumors associated with HBV-HCC were more aggressive compared to tumors associated with $\mathrm{HCV}-\mathrm{HCC}$ and $\mathrm{HCV}-\mathrm{HCC}$ was associated with more severe cirrhosis compared to HBV-HCC.

The effects of IFN on HCC are affected by several factors, including antitumor, antiviral, antiangiogenic and modulatory inflammatory factors or hepatic stellate cells in the tumor microenvironment $(12,23)$. IFN is currently the standard therapy for $\mathrm{HCV}$ infection and has also been proven to inhibit HCC development in HCV patients through clearance of HCV RNA, improving the degree of inflammation and preventing worsening of compensated cirrhosis (25). However, the preferred treatment option for the majority of patients with HBV infection is not IFN, but rather nucleotide/nucleoside analogs (NA). A previous meta-analysis demonstrated that NA treatment achieved a more profound reduction in HCC risk compared to IFN, which produced only a modest effect (78 vs. $34 \%$, repectively) (26). Since a high viral load is the most important factor leading to cirrhosis and cancer development in the liver, the more effective reduction in HCC risk may be associated with the more profound effects of viral suppression with oral antiviral agents compared to IFN.

IFN in HCV-HCC reduced both early and late recurrence. IFN reduced early recurrence by directly inhibiting tumor metastasis. Ikeda et al (27) found that IFN $\beta$ significantly reduced early recurrence of pure HCV-HCC through a direct antitumor effect. Both IFN $\alpha$ and IFN $\beta$ are type I IFNs, share the same IFN receptors and have several similar biological functions, although the direct antitumor effect of IFN $\alpha$ is weaker. However, the efficacy of IFN in reducing early recurrence of HCV-HCC may also be associated with its antiviral and anti-inflammatory effects, as microenvironmental factors (28) have been found to contribute to tumor micrometastasis and IFN may reduce early recurrence by improving the microenvironment. IFN improves liver function and relieves liver cirrhosis, which lead to a decrease in late recurrence. IFN was found to reduce transaminase levels and to improve liver function and liver cirrhosis. In the present study, liver cirrhosis was more severe in $\mathrm{HCV}-\mathrm{HCC}$ compared to that in HBV-HCC patients. The benefits of antiviral therapy for reducing late recurrence of HCC have already been accepted by several investigators, as there are data suggesting that antiviral therapy may modulate the pro-oncogenic field effect in the liver. Kubo et al (22) reported increased albumin and decreased alanine transaminase and total bilirubin levels in IFN-treated patients, indicating improved liver function. In the study by Mazzaferro et al (4), early (within 2 years) and late recurrences were analyzed separately and the only significant result was observed for late recurrences among HCV-pure patients $(\mathrm{P}=0.032)$. Huang et al (29) proved the feasibility of PEG-IFN/ribavirin therapy with current treatment guidelines in chronic $\mathrm{HVC}$ patients after successful eradication of $\mathrm{HCC}$.

IFN did not reduce recurrence rates in HBV-HCC for two main reasons. First, IFN is not the preferred antiviral treatment option for the majority of patients with HBV infection. High viral load is recognized as one of the key risk factors for HBV-HCC recurrence following resection (30) and antiviral treatment with NA has been proven to significantly decrease early recurrence of HBV-HCC (31). Since early HBV-DNA suppression with antiviral treatment improved the prognosis of patients with HBV-HCC (32) and since IFN has been reported to be more effective in responders compared to non-responders, it is important to monitor serum HBV DNA levels prior to and following surgery and/or ablation of HCC and to evaluate its association with recurrence. IFN plus NA administered in an adjuvant setting may be more promising. 
Second, there may be subgroups of patients who are sensitive to IFN therapy. Evidence has demonstrated that IFN exerts direct antitumor effects. IFN $\alpha / \beta$ exerted direct antitumor effects on HBV-HCC independent of hepatitis status and liver inflammation $(33,34)$. Lo et al $(13)$ reported that IFN was more effective in TNM stage III/IVA patients, indicating that IFN exerts direct antitumor effects. Moreover, Qian et al (35) reported that IFN only reduced tumor recurrence in patients with P48-positive tumors. More recently, Ji et al (36) observed that patients whose tumors exhibited low miR-26 expression had shorter overall survival, but a better response to IFN therapy compared to patients whose tumors exhibited a high expression of miR-26. Therefore, personalized treatment should be administered to HBV-HCC patients according to specific tumor markers, such as P48 or miR-26.

Subset analyses of individual studies suggested that IFN should be administered for longer periods of time, as it may not only reduce recurrence rates, but also increase the possibility of receiving curative treatment after tumor recurrence. In the study by Sun et al (18), during the first 18 months after resection (the planned duration for IFN $\alpha$ treatment), the recurrence rate in the control group was higher compared to that in the IFN $\alpha$ treatment group [49.2\% (58/118) vs. $36.4 \%$ (43/118)]; $\mathrm{P}=0.0485$ for $\left.\chi^{2}\right]$, but the recurrence rates after the first 18 months were not significantly different between the two groups [23.2\% (13/56) vs. 32.9\% (24/73); $\mathrm{P}=0.2292$ for $\left.\chi^{2}\right]$. Lo et al (13) used high-dose IFN for 6 months after surgery and recorded 10 recurrence events in the control group and 3 recurrence events in the IFN group during 6 months of treatment, 12 recurrence events in the control group during 12 months of treatment and 9 recurrence events in the IFN group after 12 months of treatment. Thereafter, after another 6 months, there were 12 recurrence events in the control and 12 recurrence events in the IFN group, indicating that IFN should be administered over a longer period of time.

This study has several limitations. The included studies used surgery, ablation or surgery and ablation. Furthermore, there were only a few RCTs, they were all from a single center instead of multiple centers and most of the published studies had small sample sizes. The types of IFN and IFN regimens used in the published studies varied widely regarding total dosage, average dosage per week and treatment duration. Long-term IFN, such as PEG-IFN, as well as short-term IFN, were used and certain studies used IFN $\alpha$ or IFN $\beta$ or IFN $\alpha-1 b$ and IFN $\alpha-2 b$. Further clinical trials should use a standardized IFN regimen to yield more comparable results. Other limitations are that the side effects of IFN were not investigated and the dropout ratio was not described. The side-effects of IFNa are dose-dependent and may be severe. Lo et al (13) reported that minor influenza-like side effects were so frequent that double blinding in their study was not possible. In the study by Mazzaferro et al (4), the withdrawal rate was extremely high, which may be the reason for its negative results. In addition, the quantification and monitoring of the serum levels of HBV DNA and HCV RNA were unavailable in the majority of the studies and we were unable to evaluate the association between viral inhibition and the efficacy of IFN. Finally, study-related bias was significant in certain studies.

In conclusion, our study demonstrated that the effect of adjuvant IFN following curative treatment for HCC differed between HBV-HCC and HCV-HCC; therefore, different strategies with IFN in an adjuvant setting should be used to treat HCCs with different hepatitis backgrounds. For HCV-HCC, IFN with an additional antiviral treatment, such as ribavirin, may be promising. However, in HBV-HCC, individualized therapy according to specific molecular markers may be necessary prior to IFN treatment, while antiviral drugs combinations may be required for patients with high viral loads.

\section{Acknowledgements}

This study was funded by a grant from the National Natural Science Foundation of China (no. 81101871).

\section{References}

1. Jemal A, Bray F, Center MM, et al: Global cancer statistics. CA Cancer J Clin 61: 69-90, 2011.

2. Zhong JH, Li H, Li LQ, et al: Adjuvant therapy options following curative treatment of hepatocellular carcinoma: a systematic review of randomized trials. Eur J Surg Oncol 38: 286-295, 2012

3. Poon RT, Fan ST, Ng IO, et al: Different risk factors and prognosis for early and late intrahepatic recurrence after resection of hepatocellular carcinoma. Cancer 89: 500-507, 2000.

4. Mazzaferro V, Romito R, Schiavo M, et al: Prevention of hepatocellular carcinoma recurrence with alpha-interferon after liver resection in HCV cirrhosis. Hepatology 44: 1543-1554, 2006.

5. Llovet JM, Burroughs A and Bruix J: Hepatocellular carcinoma. Lancet 362: 1907-1917, 2003.

6. Chen L, Zhang Q, Chang W, et al: Viral and host inflammationrelated factors that can predict the prognosis of hepatocellular carcinoma. Eur J Cancer 48: 1977-1987, 2012.

7. Wu JC, Huang YH, Chau GY, et al: Risk factors for early and late recurrence in hepatitis B-related hepatocellular carcinoma. J Hepatol 51: 890-897, 2009.

8. Imamura H, Matsuyama Y, Tanaka E, et al: Risk factors contributing to early and late phase intrahepatic recurrence of hepatocellular carcinoma after hepatectomy. J Hepatol 38: 200-207, 2003

9. Hoshida Y: Risk of recurrence in hepatitis B-related hepatocellular carcinoma: impact of viral load in late recurrence. J Hepatol 51: 842-844, 2009.

10. Clavien PA: Interferon: the magic bullet to prevent hepatocellular carcinoma recurrence after resection? Ann Surg 245: 843-845, 2007.

11. Oikawa T, Ojima H, Yamasaki S, et al: Multistep and multicentric development of hepatocellular carcinoma: histological analysis of 980 resected nodules. J Hepatol 42: 225-229, 2005.

12. Wang L, Wu WZ, Sun HC, et al: Mechanism of interferon alpha on inhibition of metastasis and angiogenesis of hepatocellular carcinoma after curative resection in nude mice. J Gastrointest Surg 7: 587-594, 2003.

13. Lo CM, Liu CL, Chan SC, et al: A randomized, controlled trial of postoperative adjuvant interferon therapy after resection of hepatocellular carcinoma. Ann Surg 245: 831-842, 2007.

14. Lin SM, Yu ML, Lee CM, et al: Interferon therapy in HBeAg positive chronic hepatitis reduces progression to cirrhosis and hepatocellular carcinoma. J Hepatol 46: 45-52, 2007.

15. Miyake Y, Takaki A, Iwasaki Y and Yamamoto K: Meta-analysis: interferon-alpha prevents the recurrence after curative treatment of hepatitis C virus-related hepatocellular carcinoma. J Viral Hepat 17: 287-292, 2010.

16. Shen YC, Hsu C, Chen LT, et al: Adjuvant interferon therapy after curative therapy for hepatocellular carcinoma (HCC): a meta-regression approach. J Hepatol 52: 889-894, 2010.

17. Singal AK, Salameh H, Kuo YF and Fontana RJ: Meta-analysis: the impact of oral anti-viral agents on the incidence of hepatocellular carcinoma in chronic hepatitis B. Aliment Pharmacol Ther 38: 98-106, 2013.

18. Sun HC, Tang ZY, Wang L, et al: Postoperative interferon alpha treatment postponed recurrence and improved overall survival in patients after curative resection of HBV-related hepatocellular carcinoma: a randomized clinical trial. J Cancer Res Clin Oncol 132: 458-465, 2006. 
19. Chen LT, Chen MF, Li LA, et al: Long-term results of a randomized, observation-controlled, phase III trial of adjuvant interferon alpha-2b in hepatocellular carcinoma after curative resection. Ann Surg 255: 8-17, 2012.

20. Jeong S, Aikata H, Katamura Y, et al: Low-dose intermittent interferon-alpha therapy for HCV-related liver cirrhosis after curative treatment of hepatocellular carcinoma. World $\mathrm{J}$ Gastroenterol 13: 5188-5195, 2007.

21. Kudo M, Sakaguchi Y, Chung H, et al: Long-term interferon maintenance therapy improves survival in patients with $\mathrm{HCV}$-related hepatocellular carcinoma after curative radiofrequency ablation. A matched case-control study. Oncology 72 (Suppl 1): 132-138, 2007

22. Kubo S, Nishiguchi S, Hirohashi K, et al: Randomized clinical trial of long-term outcome after resection of hepatitis $C$ virus-related hepatocellular carcinoma by postoperative interferon therapy. Br J Surg 89: 418-422, 2002.

23. Breitenstein S, Dimitroulis D, Petrowsky H, et al: Systematic review and meta-analysis of interferon after curative treatment of hepatocellular carcinoma in patients with viral hepatitis. Br J Surg 96: 975-981, 2009.

24. Zhang CH, Xu GL, Jia WD and Ge YS: Effects of interferon alpha treatment on recurrence and survival after complete resection or ablation of hepatocellular carcinoma: a meta-analysis of randomized controlled trials. Int J Cancer 124: 2982-2988, 2009.

25. Nishiguchi S, Kuroki T, Nakatani S, et al: Randomised trial of effects of interferon-alpha on incidence of hepatocellular carcinoma in chronic active hepatitis $\mathrm{C}$ with cirrhosis. Lancet 346: 1051-1055, 1995.

26. Sung JJ, Tsoi KK, Wong VW, et al: Meta-analysis: treatment of hepatitis B infection reduces risk of hepatocellular carcinoma. Aliment Pharmacol Ther 28: 1067-1077, 2008.

27. Ikeda K, Arase Y, Saitoh S, et al: Interferon beta prevents recurrence of hepatocellular carcinoma after complete resection or ablation of the primary tumor-A prospective randomized study of hepatitis $\mathrm{C}$ virus-related liver cancer. Hepatology 32: 228-232, 2000

28. Budhu A, Forgues M, Ye QH, et al: Prediction of venous metastases, recurrence, and prognosis in hepatocellular carcinoma based on a unique immune response signature of the liver microenvironment. Cancer Cell 10: 99-111, 2006.

29. Huang JF, Yu ML, Huang CF, et al: The efficacy and safety of pegylated interferon plus ribavirin combination therapy in chronic hepatitis $\mathrm{C}$ patients with hepatocellular carcinoma post curative therapies - a multicenter prospective trial. J Hepatol 54: 219-226, 2011.
30. Hung IF, Poon RT, Lai CL, et al: Recurrence of hepatitis B-related hepatocellular carcinoma is associated with high viral load at the time of resection. Am J Gastroenterol 103: 1663-1673, 2008.

31. Yin J, Li N, Han Y, et al: Effect of antiviral treatment with nucleotide/nucleoside analogs on postoperative prognosis of hepatitis B virus-related hepatocellular carcinoma: a two-stage longitudinal clinical study. J Clin Oncol 31: 3647-3655, 2013.

32. Huang G, Yang Y, Shen F, et al: Early viral suppression predicts good postoperative survivals in patients with hepatocellular carcinoma with a high baseline HBV-DNA load. Ann Surg Oncol 20: 1482-1490, 2013.

33. Yang JQ, Pan GD, Chu GP, et al: Interferon-alpha restrains growth and invasive potential of hepatocellular carcinoma induced by hepatitis B virus X protein. World J Gastroenterol 14: 5564-5569, 2008

34. Yamazaki K, Suzuki K, Ohkoshi S, et al: Temporal treatment with interferon-beta prevents hepatocellular carcinoma in hepatitis B virus X gene transgenic mice. J Hepatol 48: 255-265, 2008.

35. Qian YB, Zhang JB, Wu WZ, et al: $\mathrm{P} 48$ is a predictive marker for outcome of postoperative interferon-alpha treatment in patients with hepatitis B virus infection-related hepatocellular carcinoma. Cancer 107: 1562-1569, 2006.

36. Ji J, Shi J, Budhu A, et al: MicroRNA expression, survival, and response to interferon in liver cancer. N Engl J Med 361: 1437-1447, 2009.

37. Suou T, Mitsuda A, Koda M, et al: Interferon alpha inhibits intrahepatic recurrence in hepatocellular carcinoma with chronic hepatitis C: a pilot study. Hepatol Res 20: 301-311, 2001.

38. Miyaguchi S, Watanabe T, Takahashi $\mathrm{H}$, et al: Interferon therapy for hepatocellular carcinoma patients with low HCV-RNA levels. Hepatogastroenterology 49: 724-729, 2002.

39. Shiratori Y, Shiina S, Teratani T, et al: Interferon therapy after tumor ablation improves prognosis in patients with hepatocellular carcinoma associated with hepatitis $C$ virus. Ann Intern Med 138: 299-306, 2003.

40. Jeong SC, Aikata H, Katamura Y, et al: Effects of a 24-week course of interferon-alpha therapy after curative treatment of hepatitis $\mathrm{C}$ virus-associated hepatocellular carcinoma. World J Gastroenterol 13: 5343-5350, 2007

41. Hagihara H, Nouso K, Kobayashi Y, et al: Effect of pegylated interferon therapy on intrahepatic recurrence after curative treatment of hepatitis C virus-related hepatocellular carcinoma. Int J Clin Oncol 16: 210-220, 2011. 